\title{
Lipid Peroxidation in Dunaliella Salina IPPAS-294 Cells Modified by Ionol at High Salinity in Optimal and Low-Temperature Cultivation Conditions
}

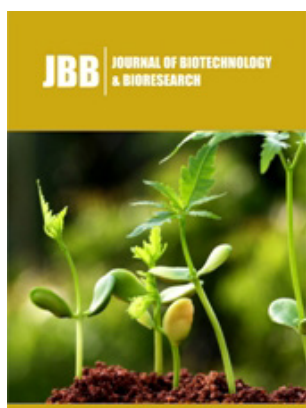

*Corresponding author: Jalilova AR, Department of Biophysics and Molecular Biology, Baku State University, Azerbaijan

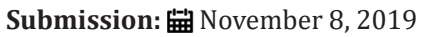

Published: 悳December 13, 2019

Volume 2 - Issue 3

How to cite this article: Jalilova $A R$ Lipid Peroxidation in Dunaliella Salina IPPAS-294 Cells Modified by Ionol at High Salinity in Optimal and Low-Temperature Cultivation Conditions. J Biotech Biores.2(3). JBB.000536.2019.

Copyright@ Jalilova AR, This article is distributed under the terms of the Creative Commons Attribution 4.0 International License, which permits unrestricted use and redistribution provided that the original author and source are credited.

\section{Jalilova AR*}

Department of Biophysics and Molecular Biology, Azerbaijan

\begin{abstract}
In this work, have been presented results of bio productivity study and quantity of formed MDA in Dunaliella cells at high salinity in optimal and low-temperature cultivation conditions. It became clear that, in conditions of low temperature stress, bioproductivity of cells decreases $25 \%$ in regard to optimal cultivating regime. The cell modification by 2,6 di-tret-butyl cresol (ionol) leads to growth stimulation of Dunaliella culture in optimal regime: $(25-350 \mathrm{mkM}) 2-7 \%$ respectively, and in conditions of low temperature stress in range of concentrations (25-350mkM) 5-8\%. It was established that, the concentrations $25-50 \mathrm{mkm} 2,6$ di-tret-butyl cresol in mineral medium cause 30-70\% increase of MDA content in cells and then $35-60 \%$ decrease of its content at concentrations $15-300 \mathrm{mkm}$ in optimal regime. In low temperature regime concentrations $25-50 \mathrm{mkm}$ of BHT, MDA quantity remains in control, but at concentrations $15-300 \mathrm{mkm}$ decreases $4-20 \%$.
\end{abstract}

Keywords: Dunaliella; Bioproductivity; Salinity; Ionol; Low temperature stress; Lipid peroxidation

\section{Introduction}

As a result of various factors such as ultraviolet radiation exposure, low and high temperatures, the presence of additives, pollutants in nutritional supplements and drinking water influencing the environment, lead to the formation of radicals. In every living organism constantly occur active forms of exogenous, peroxide, hydro peroxide and other radicals [1]. Simultaneously with the formation of these radicals, happens their destruction with the help of antioxidant enzyme system. Excess accumulation of forming concentrations over cancelled radicals leads to development of oxidative stress, which accompanies with injure of biological molecule, lipid oxidation, protein and DNA modification [2-4]. Influence of adverse temperatures on plants is one of most common stressors. So, most part of plants, during a year, is exposed to low positive temperatures, frost $[5,6]$.

ROS has a special place among stress metabolites. The important role of ROS in the launch of protective reactions to abiogenists causes no doubt. In the cultivation of seeds, at the presence of ionol, both ROS formation and especially, superoxide are strongly depressed [7]. It is clear that, as well as formation and inactivity of ROS, the antioxidant ionol can inhibit ROS-induced exit from mitochondria to cytoplasm cytochrome [8]. Significantly, reducing of oxidative stress and its consequences allows addition of synthetic antioxidants, such as ionol and its derivatives belonging to a class of hundred phenols in mineral medium. The purpose of the work is the investigation of lipid peroxidation in Dunaliella cells cultivated in optimal and low-temperature cultivation conditions at high salinity and modified by various concentrations of ionol.

\section{Materials and Methods}

As investigation object was used green algae Dunaliella Salina IPPAS-294, taken from the saline lake Masazir located on the north eastern part of Baku. The algae were grown at 27 ${ }^{\circ} \mathrm{C}$ temperature in glass photo reactors $(250 \mathrm{ml})$, in the installation for growing unicellular algae. Mineral medium contained (g/l): NaCl-175.5 (30M), $\mathrm{KNO}_{3}-50 ; \mathrm{KH}_{2} \mathrm{PO}_{4}-125 ; \mathrm{MgSO}_{4}$ $50 ; \mathrm{FeSO}_{4}-0.009$ and microelement solutions $(1 \mathrm{mg} / \mathrm{l})$. The cell suspension in photo reactors 
was irradiated by white light $\left(16 \mathrm{Wt} / \mathrm{m}^{2}\right)$ within 24 hours and permanently purged with mixture (air $+1.5 \% \quad \mathrm{CO}_{2}$ ) at $25{ }^{\circ} \mathrm{C}$ temperature in optimal and blowing of air mixture 50 (low temperature stress) to photoreactors. The culture growth rate was determined by periodically counting cell number in Qoryayev chamber under the microscope or by nephelometric measurement of optic suspension density in photoelectrocolorimeter.

The cell suspension prepared for measuring MDA content was led to 106 cells/ml (optic density $\mathrm{OD}=0.8$ ).

The evaluation degree of lipid peroxidation (POL) was carried out by the method of determining MDA content in Dunaliella Salina cells-method based on the reactions with thibarbituric acids. The cell suspension $(35 \mathrm{ml})$ was centrifuged $30000 \mathrm{rev} / \mathrm{min}$. within 10 minutes. The resulting sediment was homogenized in $20 \mathrm{ml}$ $0.1 \%$ TCA. Homogenate was centrifuged at $30000 \mathrm{rev} / \mathrm{min}$. within 10 minutes. To the $1 \mathrm{ml}$ supernatant was added $4 \mathrm{ml} 20 \%$ TCA, containing $0.5 \%$ TBA. The mixture was heated in water bath at 95 ${ }^{0} \mathrm{C}$ within 30 minutes and immediately cooled in running water. After centrifugation of mixture at 30000rev/min within 10 minutes was determined optic density of supernatant at $532 \mathrm{~nm}$ [3]. The content of MDA was calculated with extinction coefficient equal to $1.36 \times 10^{-5} \mathrm{sm}^{-1}$, after subtraction of non-specific absorption at 532 .

\section{Results and Discussions}

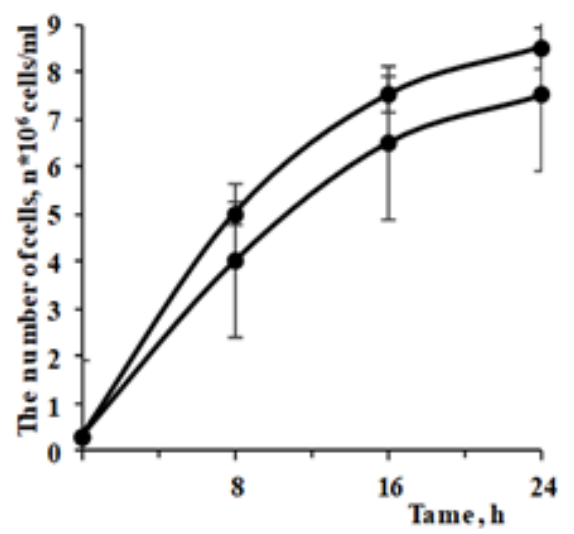

Figure 1: The growth dynamics of population in Dunaliella Salina IPPAS-294 cells in optimal

1. Low temperature

2. Cultivation regimes

Temperature 40\%, light intensity $100 \mathrm{Wt} / \mathrm{m}^{2}$

In Figure 1 (curve1), has been presented the culture growth dynamics of Dunaliella Salina IPPAS-294 microalgae in optimal conditions (temperature $27{ }^{\circ} \mathrm{C}$, light intensity $16 \mathrm{Wt} / \mathrm{m}^{2}$, content of $\mathrm{CO}_{2}$ in air mixture $1.5 \%$, mineral medium containing $30 \mathrm{MNaCl}$ ) cell cultivation in glass photo reactors $(250 \mathrm{ml})$ and submission of air mixture with temperature $25^{\circ} \mathrm{C}$ in intensive-accumulative regime of cultivation in within 24 hours has showed that, optic density of cell suspension increases 3 times. Such tendency of population growth also continues in subsequent re-options of growing control suspensions. Submission of air mixture 50 (low temperature stress) leads to growth retardation and 25\% decrease of bioproductivity (curve 2). In spite of growth dynamics, the decrease of population in low temperature stress cell deletion during 24 hourly cultivation in intensive-accumulative regime shows high rate (2-3 times increase of optic density). In these conditions, synthetic antioxidant 2,6 ditret-butyl cresol at various concentrations was added to mineral growing medium and culture growth dynamics was observed.

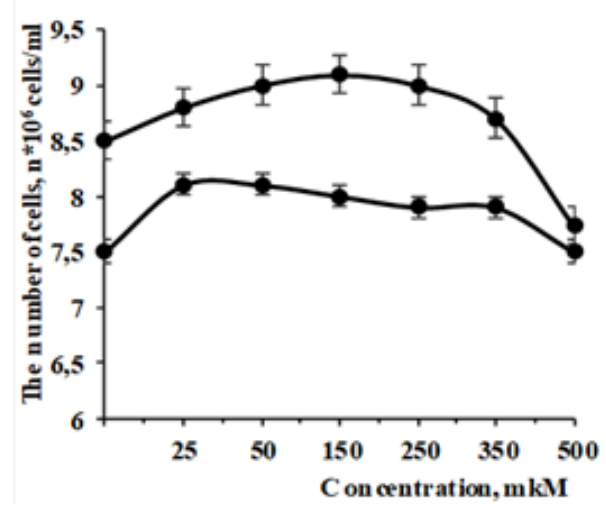

Figure 2: The growth dynamics of population in Dunaliella Salina IPPAS-294 cells in optimal

1. Low temperature

2. Cultivation regimes

Temperature $40 \%$, light intensity $100 \mathrm{Wt} / \mathrm{m}^{2}$

In Figure 2 have been presented the growth dependence of Dunaliella Salina IPPAS-294 cells in intensive-accumulative regime of cultivation from various concentrations of 2,6 di-tretbutyl phenol in mineral medium $(30 \mathrm{MNaCl})$. As seen in the Figure 2 , in optimal (1) and low temperature (2) cultivation regimes significantly effects on culture growth. So, at concentrations of 25$350 \mathrm{mkm}$ in mineral medium at concentrations $25-350 \mathrm{mkm}$ of 2,6 di-tret-butyl cresols in optimal cultivation regime, was observed $2-7 \%$ stimulation of cell culture growth dynamics compared to control suspensions. Thus, at these concentrations of 2, 6 di-tretbutyl cresols is comparable to activity of common phytohormones [1]. While increasing the content of 2, 6 di-tret-butyl cresols up to $500 \mathrm{mkm}$ in mineral medium, it gets a reverse sign, was observed 9\% suppression of culture growth during 24 hourly cultivation in intensive-accumulative regime. Under this influence of this antioxidant, maximum differentiation was observed at concentrations $150 \mathrm{mkm}$ (7\%) compared to control cells.

Comparable investigation of the dependence of culture growth of Dunaliella Salina IPPAS-294 cells from various concentrations of BHT in mineral medium, in conditions of low temperature stress showed that, its presence in cultivation medium effects on culture growth (Figure 2, curve2). So, in the range of concentrations 25$350 \mathrm{mkm}$ of ionol in mineral medium, was observed stimulation of culture growth, which increases control cell suspensions 5-8\%. The increase of ionol concentrations up to $500 \mathrm{mkm}$ in mineral medium, no growth stimulating action was observed and defined at the level of $100 \mathrm{~V}$. In this experiment was seen that, the presence 
of various concentrations of 2,6 di-tret-butyl cresol in mineral medium in the range of $25-350 \mathrm{mkm}$ does not affect (no growth suppression was observed) on the algae bioproductivity. In this case, cell tolerance against antioxidant increases compared to the cells, grown in optimal cultivation regime, probably, related to work of cell endogen antioxidant system and 2,6 di-tret-butyl cresols [9]. Expressed growth stimulating BHT activity at concentrations 25$350 \mathrm{mkm}$ in mineral medium in optimal cultivation regime and in the range of concentrations $25-500 \mathrm{mkm}$ in low temperature stress make this antioxidant perspective and effective for being available and reliable for regulation of culture growth in Dunaliella Salina IPPAS-294 cells.

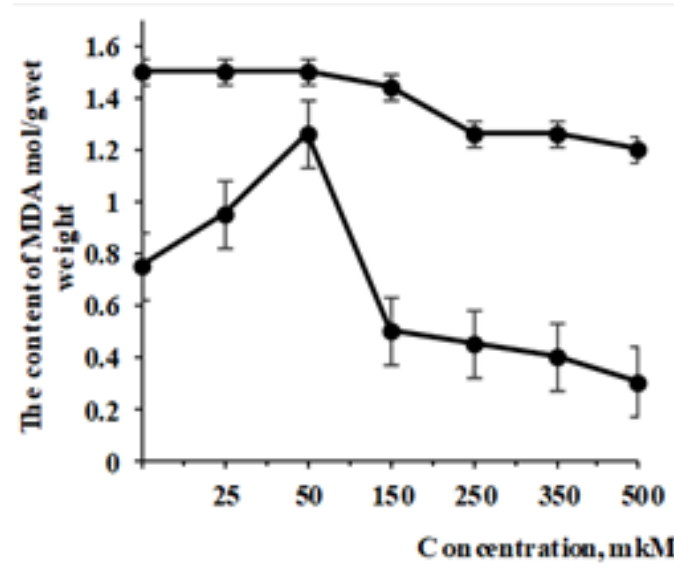

Figure 3: The dependence of MDA content in Dunaliella Salina IPPAS-294 cells from various concentrations of ionol in mineral medium $(30 \mathrm{MNaCl})$ in optimal

1. Low temperature

2. Cultivation regimes

Temperature $27 \%$, light intensity $16 \mathrm{Wt} / \mathrm{m} 2$

In Figure 3 have been presented, the growth dependence of MDA content in cells, grown in in optimal (1) and low temperature (2) cultivation regimes from various concentrations of BHT in mineral medium. As seen in the Figure 3, various concentrations of synthetic antioxidant BHT effects on MDA content in cells. So, in optimal cultivation regime (Figure 1, curve 1) in the range of 25$50 \mathrm{mkm}$, was observed increase up to $30-60 \%$ in quantity of MDA content in cells [10]. Further increase of BHT concentrations leads to MDA decrease and more effective suppression of POL process (35-60\%) in cells. Comparative investigation of quantitative indicators of MDA content in conditions of low temperature stress and at concentrations of 2,6 di-tret-butyl cresol showed that, in spite of reactive oxygen species (low temperature stress), last one actively suppresses POL process $[11,12]$. In these conditions BHT concentrations $150-500 \mathrm{mkm}$ suppress MDA content in cells 4-20\%. Only concentrations 25 and $50 \mathrm{mkm}$ do not effect on the level of lipid peroxidation in conditions of low temperature stress (Figure 3, curve 2). So, 24 hourly modification of Dunaliella Salina IPPAS-294 cells by 2,6 di-tret-butyl cresol decreases the quantity of reactive oxygen species which causes the decrease of lipid per oxidation process and finally algae bioproductivity.

\section{References}

1. Shorning B, Poleshchuk SV, Gorbatenko I, Vanyushin BF, Izvestiya A (1999) Ser Biol pp. 30-38.

2. Gaté L, Paul J, Ba GN, Tapiero H, Tew KD (1999) Oxidative stress induced in pathologies: The role of antioxidants. Biomed Pharmacother 53(4): 169-180.

3. Matés JM (2000) Effects of antioxidant enzymes in the molecular control of reactive oxygen species toxicology. Toxicology 153(1-3): 83-104.

4. Namiki M (1990) Antioxidants/antimutagens in food. Crit Rev Food Sci Nutr 29(4): 273-300.

5. Reynolds MP, Ortiz-Monasterio J, McNab A (2007) Application of physiology and wheat breeding. International Maize and Wheat Improvement Center, Mexico, pp. 254-278.

6. Trunova TI (2007) plants and low-temperature stress/64th Timityazev Nauka pp. 54.

7. Harman D (1992) Free radical theory of aging. Mutat Res 275(3-6): 257266.

8. Abdullaeva TM, Magomedova MA (2007) Plant Ecology 4: 44-47.

9. Alizadeh GI, Jalilova AR, Aliyev II, Magerramova HH (2017) Functional activity and UV-B tolerance of Dunaliella cells modified with synthetic antioxidants under low-temperature stress. News of AMEA 72(2): 106113.

10. Alizadeh GI, Jalilova AR, Maharramova K, Aliyev II (2016) The stability of functional activity in Dunaliella cells against the acute doses of UV-B irradiation, modified by synthetic antioxidants. European Journal of Biotechnology and Bioscience 4(10): 34-38.

11. Bolwell GP, Blee KA, Butt VS (1999) Free Radical Res 31: 137-145.

12. Torres MA, Jones DG, Dange YJ (2006) Plant Physiol 141: 373-378.

For possible submissions Click below: 